\title{
Complutum
}

ISSN: 1131-6993

\section{Arquitecturas salvajes. Etnoarqueología de las viviendas nilo-saharianas de Etiopía Occidental}

\author{
Álvaro Falquina ${ }^{1}$
}

Recibido: 31 de julio de 2016 / Aceptado: 23 de marzo de 2017

Resumen. Se lleva a cabo un análisis etnoarqueológico de las viviendas de las comunidades nilo-saharianas de Etiopía Occidental. El objetivo es mostrar que las casas de estas comunidades tienen un modelo espacial común, sobre el que se han desplegado diferentes variantes en la configuración interior de las casas a lo largo de su historia, incluso dentro de un mismo grupo étnico. Este modelo espacial común se materializa un tipo de casa que ordena las acciones y prácticas sociales de humanos y cosas siguiendo los principios fisiológicos del cuerpo humano.

Palabras clave: Espacio doméstico; cuerpo; agencia; sociedades no jerarquizadas; Etiopía Occidental.

\section{[en] Savage Architectures. Ethnoarchaeology of Nilo-Saharan Houses of Western Ethiopia}

\begin{abstract}
An ethnoarchaeological analysis of house buildings in Nilo-Saharan communities of Western Ethiopia is presented. The goal is to show that houses in these communities have a common spatial layout, and that several variations of their inner distribution have been implemented throughout their history, even within a single ethnic group. This common spatial layout is materialized in a type of house that orders actions and social practices involving human and things according to the physiological principles of the human body.
\end{abstract}

Keywords: Domestic Space; Body; Agency; Non-Hierarchical Societies; Western Ethiopia.

Sumario. 1. Introducción. 2. La vivienda nilo-sahariana: la casa como cuerpo. 3. Conclusión.

Cómo citar: Falquina, A. (2017): Arquitecturas salvajes. Etnoarqueología de las viviendas nilo-saharianas de Etiopía Occidental. Complutum, 28(2): 417-430.

\section{Introducción}

Las tierras de frontera entre Etiopía y Sudán históricamente han conformado un territorio con una cierta homogeneidad cultural, habitado por una amplia variedad de comunidades étnicas con una organización sociopolítica no estratificada, que han sido definidas como sociedades de frontera o deep rural (James 1980; Jedrej 1995, 2004). Este último término fue acuñado para describir las estrategias de resistencia de una serie de grupos del África Occidental que optaban por mantenerse en los márgenes del Estado (Last 1979; Mabogunje y Richards 1985; Fanthorpe 1998), frente a los campesinos que por definición son comunidades subordinadas dentro del marco estatal (Wolf 1975 [1966]).

Las comunidades deep rural se caracterizan por el conservadurismo cultural y por tratar de evitar ser sometidas por poderes externos, para lo que pueden desplegar diferentes estrategias de resistencia. Pero al igual que las comunidades campesinas, poseen un ethos comunal y una fuerte economía moral que impugna la desigualdad entre grupos familiares, insistien-

\footnotetext{
Arqueólogo

E-mail: falquina@gmail.com
} 


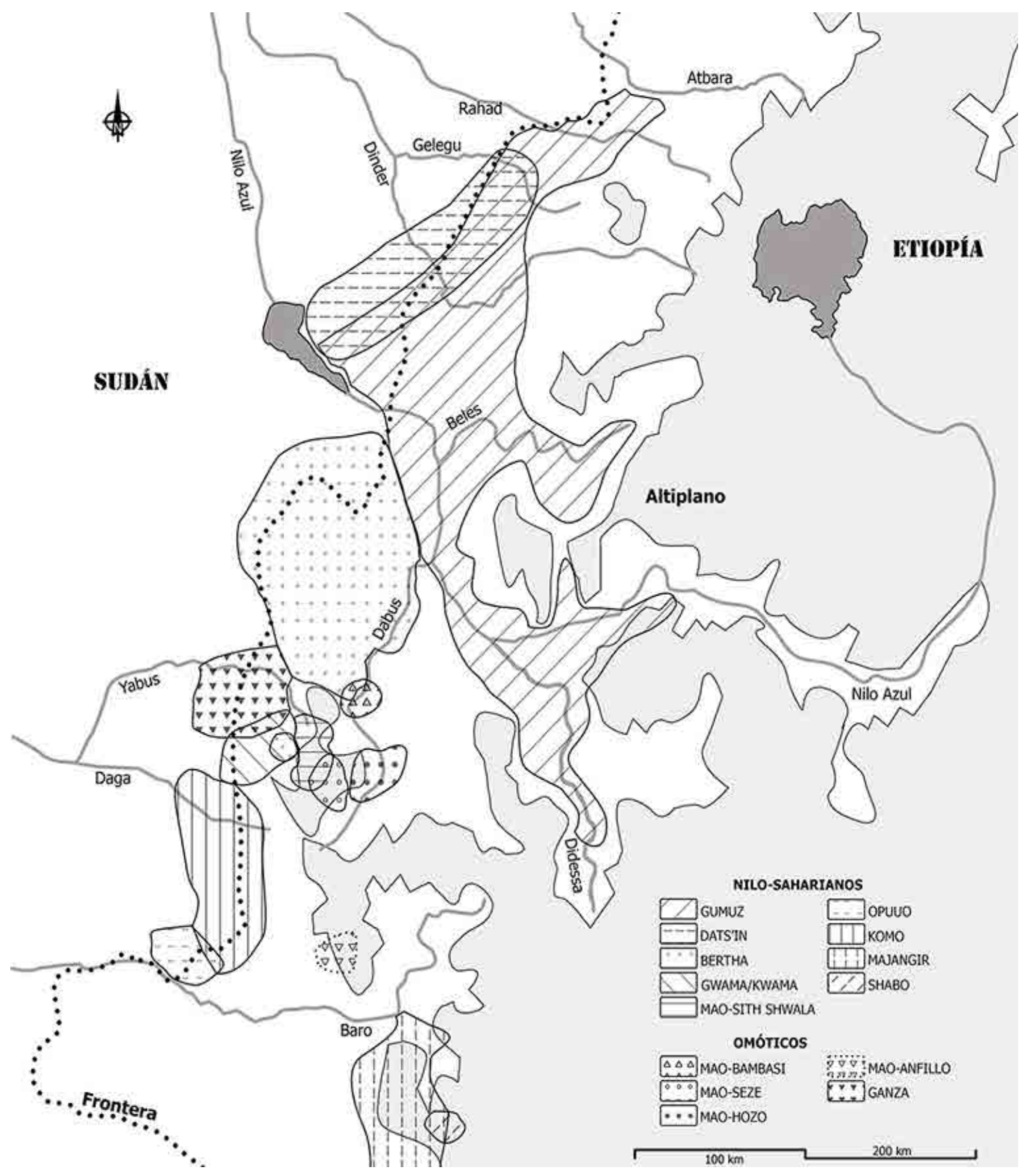

Figura 1. Mapa de distribución de las sociedades de resistencia de Etiopía Occidental. Basado en diversos planos de Wendy James y Alfredo González-Ruibal, así como en los datos de nuestro trabajo de campo.

do en la no jerarquización, la reciprocidad y la ayuda comunitaria (González-Ruibal 2014: 68 y ss.).

Existen diferencias étnicas y lingüísticas en este mosaico de grupos de Etiopía Occidental (Fig. 1). Sin embargo, este trabajo se enfoca exclusivamente en las comunidades komo, gwama/kwama, opuuo, gumuz, daats'ín y bertha, debido a que forman un subgrupo cultural definido dentro del panorama de sociedades no jerarquizadas de Etiopía Occidental. Todos estos grupos pertenecen a la familia lingüística nilo-sahariana. Pueden definirse genéricamente como agricultores de roza y quema con tecnología de palo cavador o azada, aunque siguen siendo importantes la caza, la pesca, la apicultura y la recolección. Esto quizás se deba a que sus probables orígenes se encuentran en 
poblaciones mesolíticas y neolíticas del Sudán central desplazadas hacia el sur ( $c f$. Haaland 1992; Fernández 2003).

$\mathrm{Su}$ organización social se basa en gran medida en el parentesco. Son sociedades segmentarias con un sistema matrimonial por intercambio exogámico restringido, siendo predominantes el intercambio de hermanas y el matrimonio entre primos (Grottanelli 1948; Delmet 1979; James 1975, 1986). La presión del Estado o de las religiones islámica y cristiana hacen que en algunos contextos existan otras formas matrimoniales que podrían implicar la jerarquización social entre grupos familiares patrilineales (riqueza de la novia, dote). Sin embargo, esta ausencia de estratificación sociopolítica implica la subordinación de las mujeres en estas sociedades patriarcales. A pesar de ello, la mayoría siguen teniendo elementos matrifocales (Grottanelli 1948; Delmet 1974; James 1993) anclados en una tradición cultural profundamente enraizada en los milenios precedentes (Ehret 2008: 207-19).

El desarrollo histórico de estos grupos se ha visto afectado por dos aspectos fundamentales. Por un lado, su existencia se ha relacionado con la siempre presente amenaza y agresión por parte de formaciones estatales o jerarquizadas. Este tipo de sociedades complejas han tenido su principal desarrollo desde la prehistoria en dos focos de esta región de África: el Sudán central y septentrional y el altiplano etíope (González-Ruibal 2012). Por otro lado, y en consecuencia, entre ambos territorios se fue conformando históricamente una zona de frontera, un área cultural estrechamente vinculada a una geografía particular, definida principalmente por el imponente escarpe que marca la transición entre el altiplano abisinio y las llanuras sudanesas. Tanto desde la perspectiva islámica sudanesa - dar al-harb: "tierra de guerra"- como desde la cristiana etíope -bäräha: "desierto"- esta zona fronteriza se ha concebido como un territorio peligroso al margen de la civilización, habitado por poblaciones salvajes (Donham 1986: 12; Jedrej 2004: 712-13).

Gracias en gran medida a su abrupta y a menudo difícilmente penetrable geografía, el área habitada por estas comunidades ha supuesto un refugio para ellas. Los diversos ecosistemas que conforman las tierras del escarpe han desempeñado un importante papel como espacios de protección y vitalidad para los grupos que las habitan, convirtiéndose este entorno en un actor destacado (González-Ruibal 2014:
47-53) en el mantenimiento de una economía política "salvaje" fundamentada en la no separación de sociedad y poder en dos esferas diferenciadas, si seguimos a Clastres (1987: 109-16). En adelante se utilizarán los términos "salvaje" y "primitivo" siguiendo las nociones desarrolladas por Lévi-Strauss (1997 [1964]) y Clastres (1987), frente a la tradición evolucionista propia de la antropología colonial iniciada por Lewis H. Morgan (1987 [1877]).

Además del entorno medioambiental, el entorno construido -junto con otros elementos de cultura material- también ha jugado un papel clave en el mantenimiento y desarrollo de estas sociedades no jerarquizadas, participando en y movilizando sus diferentes estrategias materiales de resistencia (González-Ruibal 2014). En este texto se trata de mostrar, además, que la concepción del espacio construido y las agencias materiales puestas en funcionamiento en los contextos domésticos nilosaharianos son coherentes con la racionalidad propia de las sociedades primitivas. Como argumenta Criado (1995: 92-93), en el "pensamiento salvaje" se establece una relación participativa del orden cultural en la naturaleza, donde el primero es una realidad distinta pero dependiente de la segunda, guiándose en gran medida por los principios del orden natural. La vivienda nilo-sahariana encarna un modelo de casa social que se ajusta muy bien a estos principios.

\section{La vivienda nilo-sahariana: la casa como cuerpo}

Existen diferentes modelos de espacios domésticos que podríamos decir normativos para cada grupo étnico. Esto no impide que dentro de un mismo grupo existan a su vez variantes regionales, derivadas en gran medida de las hibridaciones a causa del contacto y la convivencia interétnicos a lo largo de la historia. Tanto la diversidad de modelos como las hibridaciones se corresponden, por un lado, con las diferentes estrategias de resistencia desplegadas a lo largo de siglos de contacto con sociedades dominadoras (vid. González-Ruibal 2014), pero también con las experiencias de contacto entre las propias sociedades no jerarquizadas. Sin embargo, es posible apreciar en los diferentes registros de las viviendas que toda esta variabilidad se desarrolla en torno a un patrón cultural común. 

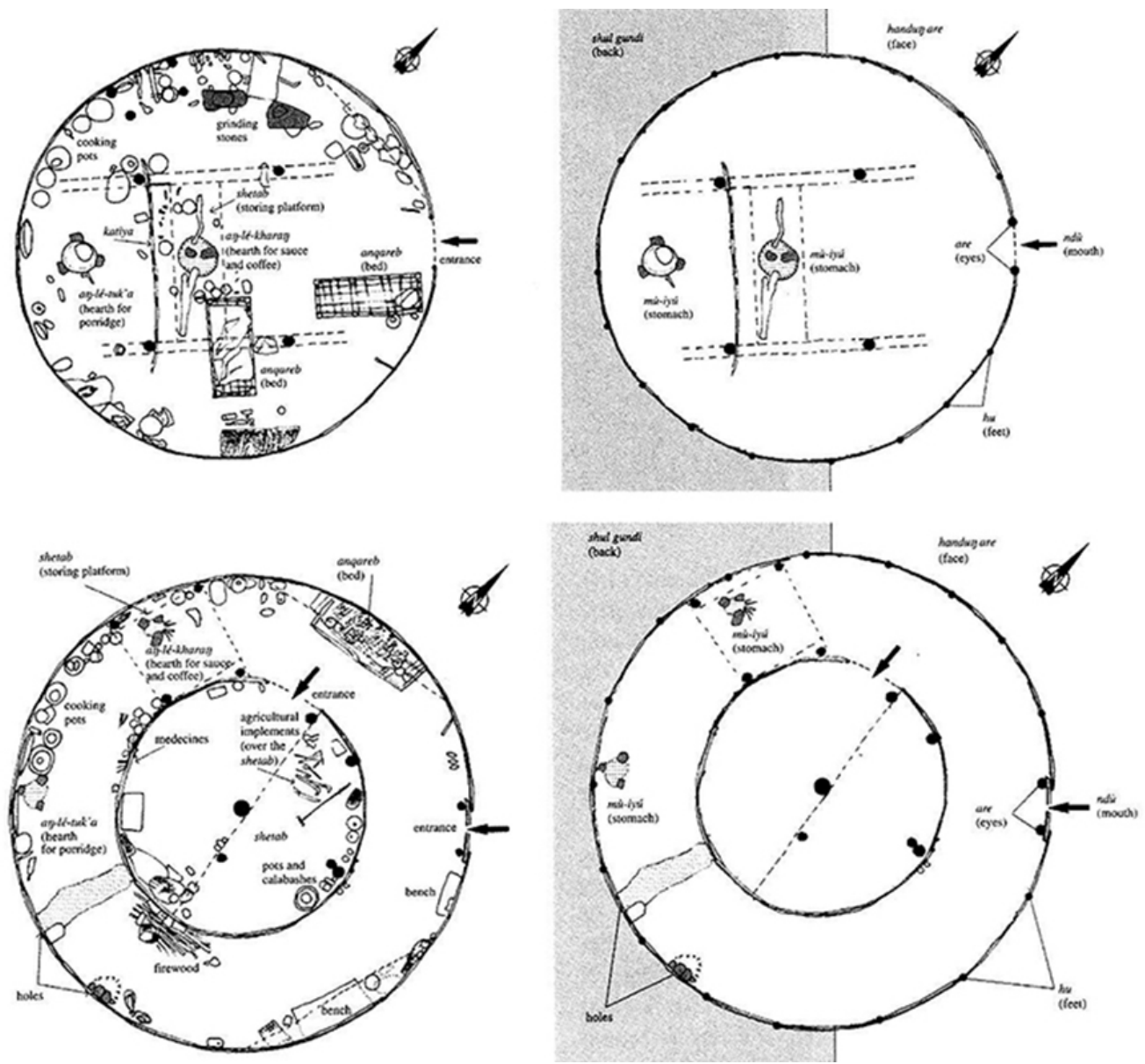

Figura 2. División del espacio interior de la casa bertha, según González-Ruibal (2006, figs. 8 y 9).

Hasta el momento, sólo entre los bertha de Benishangul se ha reconocido la existencia de un modelo común de espacio doméstico que subyace en las diferentes variantes regionales de sus viviendas (González-Ruibal 2006). Parece que todas ellas reproducen un modelo de división simbólica del espacio, el cual contrapone la parte delantera de la vivienda a la trasera. El área exterior delantera es donde se llevan a cabo la mayor parte de las actividades cotidianas, además de ser el espacio para la socialización y estar relacionado con la noción de limpieza. En cambio, en la zona tras la casa no tiene lugar ninguna actividad, y se relaciona con la suciedad, el desecho y la muerte. Se establece la misma división conceptual entre delante y detrás para el interior de la vivienda (Fig. 2).

Si consideramos la vivienda como un artefacto que funciona siguiendo unos principios prácticos dentro de un sistema de agencias, antes que como signo dentro de un sistema de comunicación (Warnier 2006: 187), debemos tratar de ver los modos concretos de acción que el edificio pone en funcionamiento y cómo afectan estos a las personas que en él residen. Siguiendo esta idea, para el análisis que nos ocupa es de gran ayuda la descripción que los propios bertha hacen de sus viviendas, en vez de entenderlas desde unas categorías analíticas dicotómicas.

Este grupo entiende su vivienda como un cuerpo humano, como explica González-Ruibal (2006: 392-93). Nombran diferentes zonas y elementos de la estructura con partes del cuerpo. La fachada es la cara, con los postes de la puerta como ojos y la propia abertura como la boca. Los postes que sostienen la pared circular de la casa son los pies. La parte trasera exterior es la espalda y el tejado, la cabeza. Finalmente, en el interior se encuentran los fuegos para cocinar, que son el estómago. Lo más interesante de esta relación entre vivienda y cuerpo es que no es principalmente meta- 
fórica, ya que la estructura realiza funciones fisiológicas, puesto que tiene órganos además de miembros.

El carácter fisiológico de sus acciones se pone definitivamente de manifiesto debido a la existencia de un "órgano de expulsión" de substancias corporales, que toma la forma de dos aberturas en la parte trasera de la pared de la estructura (Fig. 3). Éstas conectan interior y exterior en la parte posterior de la vivienda, tras la cocina, donde se encuentra el estómago de la casa. Entre los bertha, las mujeres menstruantes son confinadas en el interior de la casa y deben orinar en estos orificios, así como lavarse sobre ellos durante los siete días posteriores. Las relaciones sexuales son igualmente peligrosas tanto para los hombres como para las mujeres, por lo que deben tenerse siempre en el interior de la casa. Al finalizar, ambos deben lavar con agua sus órganos sexuales sobre estos mismos agujeros (ibid.: 393).

Así, los orificios parecen funcionar como un sistema excretor de la vivienda, de manera que se expulsen las substancias potencialmente dañinas para el interior de la casa-cuerpo y sus habitantes, alejando los peligros de polución y enfermedad, y restableciendo de este modo el equilibrio fisiológico de la casa como organismo.

Los bertha revelan el carácter procesual y práctico de la vivienda, que realiza acciones similares a las del cuerpo humano. En este sentido, la división entre la parte delantera y la trasera en el exterior no significa ni funciona igual que la misma división en el interior de la estructura. Warnier (2006) ha subrayado la importancia de esta diferencia entre dentro y fuera para la comprensión de un sistema de agencias basado en el cuerpo y sus conductas.

Si se analiza la distribución del interior de la casa-cuerpo bertha (Fig. 4), se pone de manifiesto que efectivamente existe una división entre la zona anterior y la posterior. Pero ésta no se corresponde con la división que se establece para el exterior, entre otras cosas porque ni las funciones de las dos mitades del espacio interior son las mismas que en el exterior, ni se llevan a cabo las mismas actividades. Mientras la zona exterior frente a la puerta es un espacio de expresión y socialización comunitaria, la interior delantera es fundamentalmente un "área de descanso". Allí se encuentran las camas, la ropa se cuelga en cuerdas o reposa sobre las camas, se guardan los útiles agrícolas y las armas o se dejan los zapatos. Cuerpos,

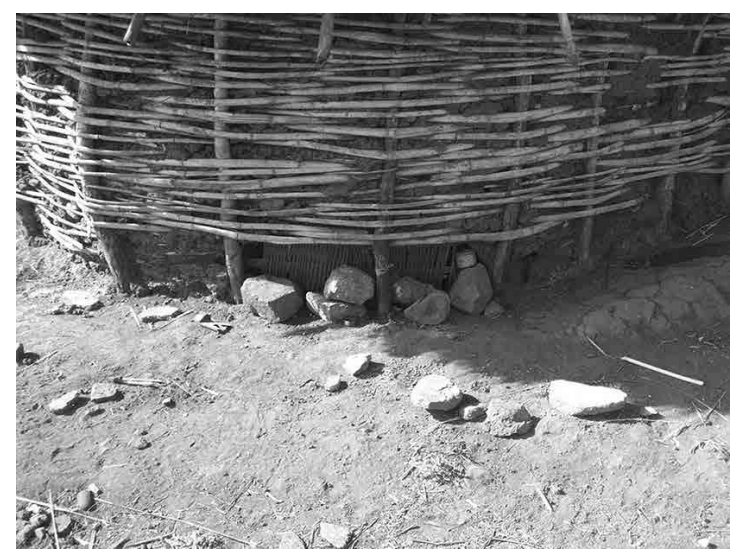

Figura 3. Detalle de los orificios u "órgano excretor" de la casa bertha, Obora (Menge wereda). Fotografía del autor.

elementos de atuendo y herramientas detienen su actividad en este espacio. Como espacio de reposo, también es el lugar donde se alimentan sus habitantes, junto a la boca de la casa.

En la zona trasera exterior no se realiza ninguna actividad. Es el lugar donde se deposita la basura, donde se sitúan las letrinas o donde se entierra a los niños menores de un año. Es un espacio para la deposición de los "restos" de la casa como organismo colectivo. En cambio, la parte posterior del interior doméstico es el espacio de mayor actividad cotidiana y ésta es exclusivamente femenina. Esta área está destinada al procesado y transformación de los alimentos. Aquí se sitúan los molinos; los fuegos y la vajilla para cocinar las gachas y las salsas, así como para preparar el café; las cerámicas y utensilios para preparar, fermentar y almacenar la cerveza; o los recipientes para guardar el agua diariamente. No extraña entonces que los bertha sitúen literalmente en esta parte el estómago de la casa. Esta zona es la que se ha denominado "área de transformación".

Como hemos visto, el interior de la vivienda funciona orgánicamente como el interior del cuerpo humano en situación de reposo, sin la actividad proyectada en el exterior colectivo. El interior de la casa-cuerpo es un espacio frágil y muy susceptible a la polución, que debe ser protegido y fortalecido por medios mágico-rituales (González-Ruibal 2006: 39394) al igual que el cuerpo de las personas. La casa puede caer enferma ante el ataque de un espíritu maligno. Es en este marco donde mejor se entienden tanto el apotropaísmo como las prohibiciones y normas -las más destaca- 

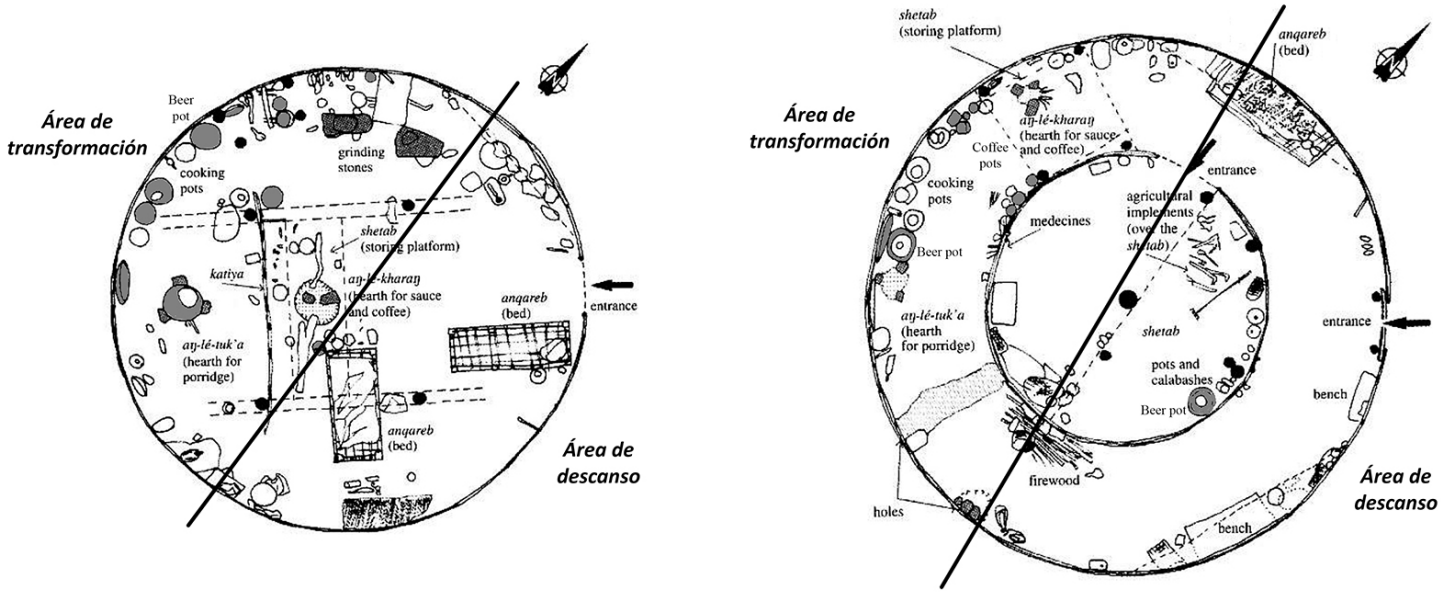

Figura 4. División interior de la casa bertha en "área de descanso" y "área de transformación", sobre planos de González-Ruibal (2006, figs. 8 y 9).

das en torno a la menstruación y la sexualidadque rodean al interior de la casa bertha.

Entre los demás grupos nilo-saharianos de Etiopía Occidental, no hay una relación metafórica entre vivienda y cuerpo como la que establecen los bertha. Sin embargo, la distribución de las áreas de actividad, tanto en el interior como en el exterior, es compartida por la mayoría de ellos. Al analizar el interior de las casas de gumuz, daats'iin, gwama-kwama, komo y opuuo es posible establecer la misma división que en la casa bertha, entre la parte delantera como "área de descanso" y la trasera como "área de transformación", a pesar de la existencia de diferentes elementos estructurales para cada grupo y de las variaciones regionales.

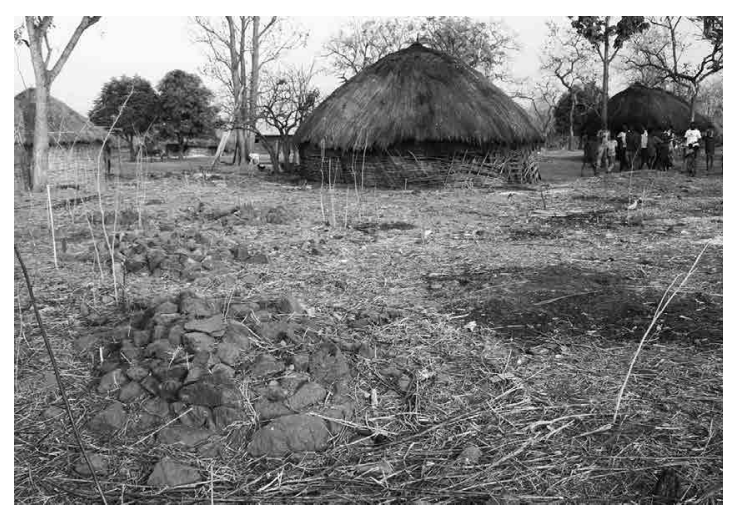

Figura 5. Localización de tumbas tras las casas. Poblado gumuz de Lugo (Kamashi). Fotografía de Alfredo González-Ruibal.
Gumuz y daats'íin no comparten el mismo concepto de limpieza que los grupos con lenguas koman (komo, opuuo y gwama-kwama) o los bertha. Éstos ponen un gran cuidado en la limpieza y barrido de las áreas exteriores frente a sus casas, donde se realizan la mayoría de las actividades, tienen lugar las fiestas colectivas y se localizan la mayor parte de las estructuras del conjunto doméstico (corrales, secaderos, graneros, casas de invitados). Los conjuntos gumuz, en cambio, son más sucios y desordenados (González-Ruibal 2014: 148), al igual que los daats'íin. Pero el área ante la puerta de la casa se diferencia de la parte trasera porque es un espacio de tierra pisada donde también se realizan la mayor parte de las actividades cotidianas, se congregan familiares y

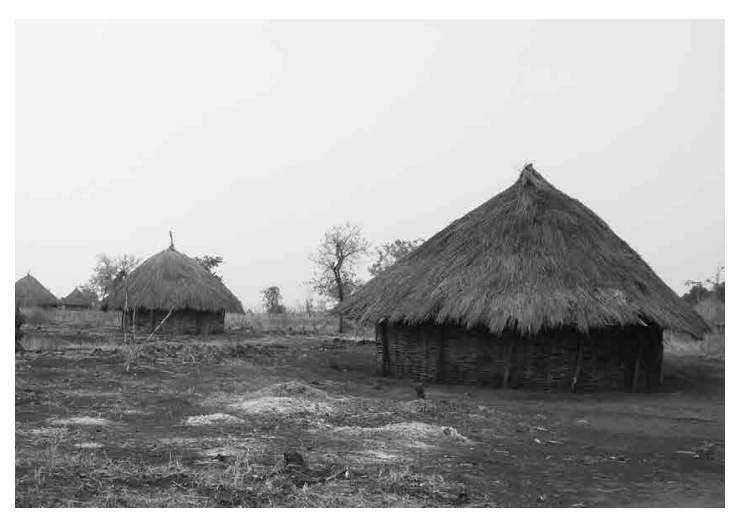

Figura 6. Cenizales tras una casa gwama en el poblado de Benishuba (Tongo-Begi special wereda). Fotografía de Xurxo Ayán. 

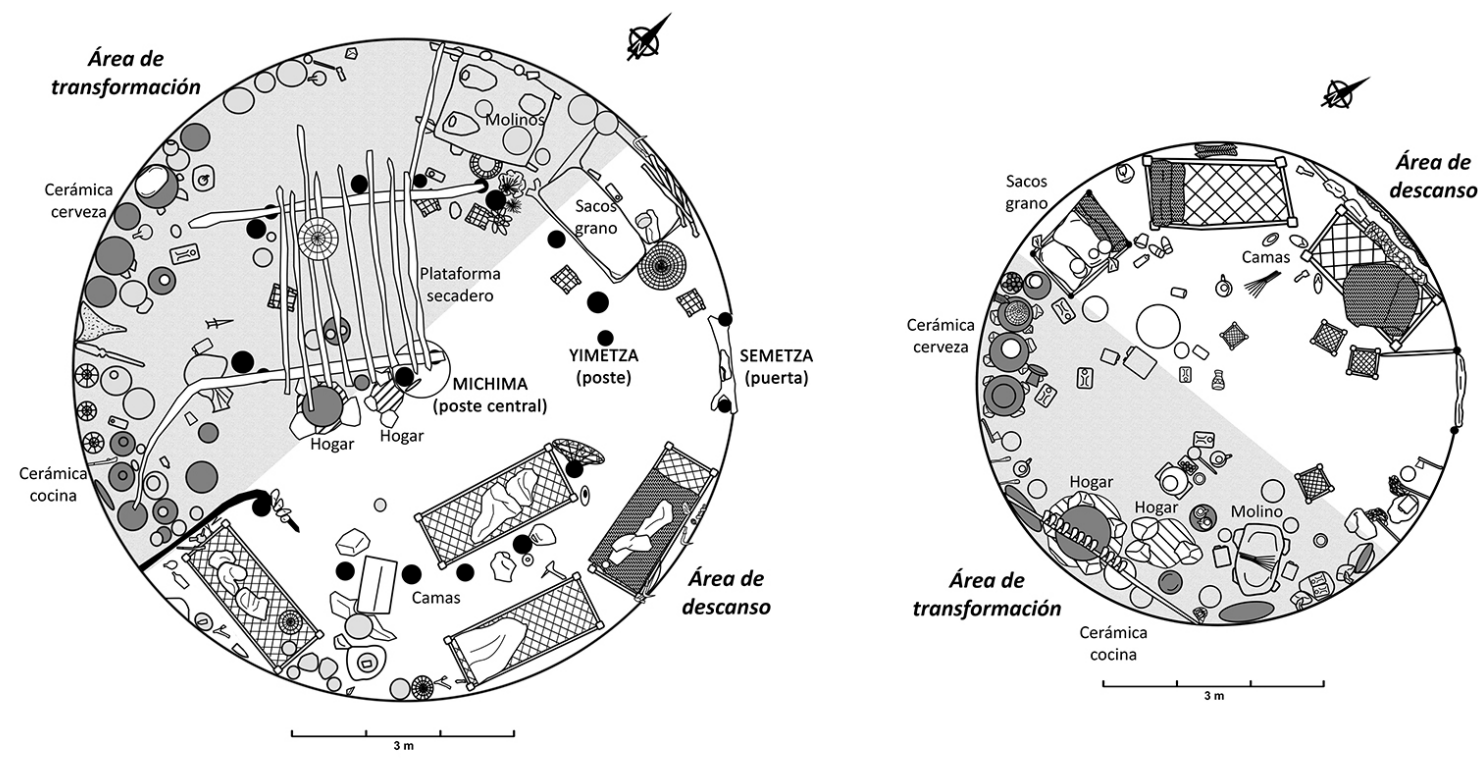

Figura 7. División del espacio interior de las casas gumuz -izquierda- (Bowla Dibatsa, Metekel -sobre plano de González-Ruibal et al. 2009: fig. 4) y daats'ín -derecha- (Omedla, Qwara-plano del autor).

vecinos diariamente o con ocasión de alguna celebración, y se distribuyen el resto de estructuras que conforman el conjunto doméstico.

Por otra parte, todos los grupos nilo-saharianos comparten la misma concepción simbólica del área situada tras la casa. Como veíamos, los bertha la asocian con nociones de suciedad, desecho y muerte. Gumuz, daats'íin y koman la conciben del mismo modo. Es un espacio donde crecen las cosechas o que está cubierto de rastrojo, donde hay restos de actividades y de antiguas construcciones, donde se hacen las deposiciones o donde pueden verse distribuidas tumbas de adultos y niños (Figs. 5 y 6$)$.

En cuanto al interior, las viviendas gumuz y daats'íin son muy similares. No en vano, están emparentados lingüísticamente (Ahland "en prensa".). Si bien es cierto que las casas gumuz normalmente tienen un poste central que habitualmente se rodea de un círculo concéntrico de postes interiores que soportan el tejado (donde habitualmente se sitúa la plataforma de almacenaje y secado de la cosecha), hemos observado que en las zonas de Metema y Qwara, el interior es completamente diáfano, al igual que en el caso de los daats'íin, con quienes conviven en esta última región.

En cambio, la distribución de las áreas de actividad dentro de la casa es similar: ambos grupos dividen el espacio interior en un área de descanso de personas y cosas, y un área de acti-

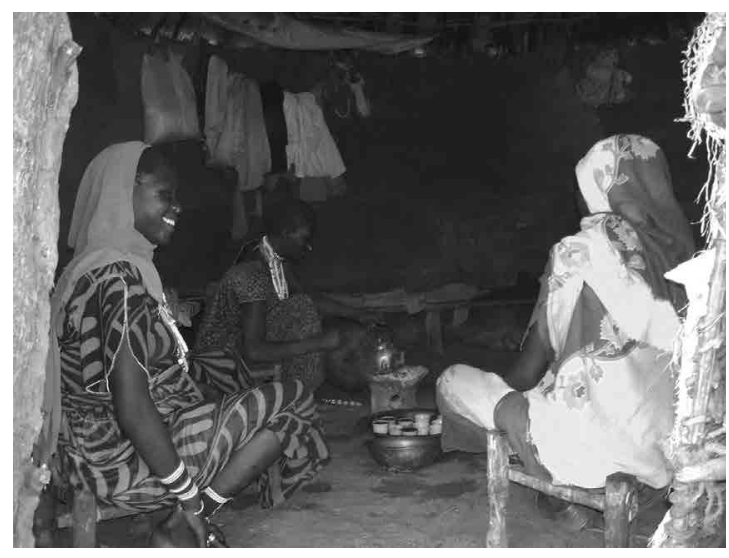

Figura 8. Mujeres gumuz tomando café junto a la puerta de la casa (Aftit, Metema). Fotografía del autor.

vidad destinada a la transformación de alimentos, con las mismas características descritas para la casa bertha (Fig. 7). En la zona anterior, el área localizada ante la puerta es el espacio de sociabilidad doméstico, donde se sitúan las banquetas para las reuniones cotidianas, para comer colectivamente, para tomar café, donde las mujeres se congregan para confeccionar adornos corporales, o donde los niños se reúnen para hacer música (Fig. 8). Como en el caso de los bertha, también es el espacio para el descanso de personas, de almacenamiento de los sacos de grano, donde se cuelgan de la 


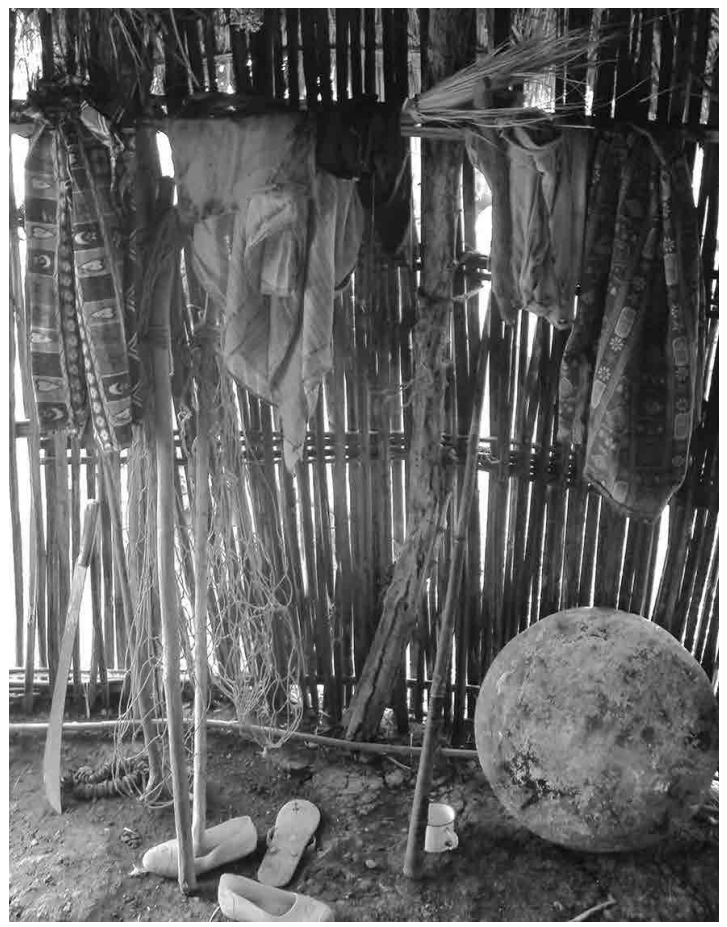

Figura 9. Objetos -ropa, yindigha, machete, calzado-reposando en el "área de descanso" de una casa daats'íin (Omedla, Qwara). Fotografía del autor.

pared los útiles agrícolas, donde se depositan sobre el suelo los instrumentos para acarrear agua o leña por parte de las mujeres o donde se guardan los arcos y flechas de los hombres (Fig. 9). En contraste, la zona trasera es donde se sitúan los fuegos para cocinar y preparar la pasta para hacer cerveza, las plataformas para los molinos o donde se localizan las cerámicas de cocina y fermentación de la cerveza, apoyadas sobre la pared.
Entre los gwama-kwama también existen diferencias en cuanto a los elementos estructurales utilizados en el interior de la vivienda. El espacio central no lo ocupa un poste, pero nunca es diáfano. Generalmente está ocupado por una estructura cuadrangular hecha con cuatro postes que sujetan la plataforma para el almacenaje y secado de la cosecha, algo que recuerda a la estructura concéntrica propia de los gumuz o algunos modelos documentados entre los bertha, por ejemplo, en la aldea de Obora (Menge). Lo que sí diferencia el modelo gwama-kwama de los demás es la existencia de una pared hecha de postes y bambú que separa la zona de la entrada -destinada a los visitantes, los animales y al dormitorio de los niños- de la zona posterior -donde se sitúan las camas de los progenitores, la plataforma y el área de cocina. Esta pared no coincide con la división que, en el caso de este grupo, también puede establecerse entre "área de descanso" y "área de transformación" (Fig. 10).

A diferencia del resto de grupos nilo-saharianos, komo y opuuo tienen un modelo de vivienda que se ha descrito como casa "exteriorizada" (Fig. 11), en el que el espacio doméstico está formado por diversas cabañas con funciones diferenciadas (casa de invitados, dormitorios, cocina) dentro de un espacio abierto, muy cuidado y pisado, delimitado por campos de cultivo o por el bosque (GonzálezRuibal et al. 2013: 72-3). Este tipo de conjunto doméstico aparentemente difiere del resto de viviendas nilo-saharianas, pero en él también puede establecerse la división entre área delantera y área trasera definida por las mismas características que segmentan las casas en el resto de grupos (Figs. 12 y 13). Aquí, interior $\mathrm{y}$ exterior se confunden. La parte delantera
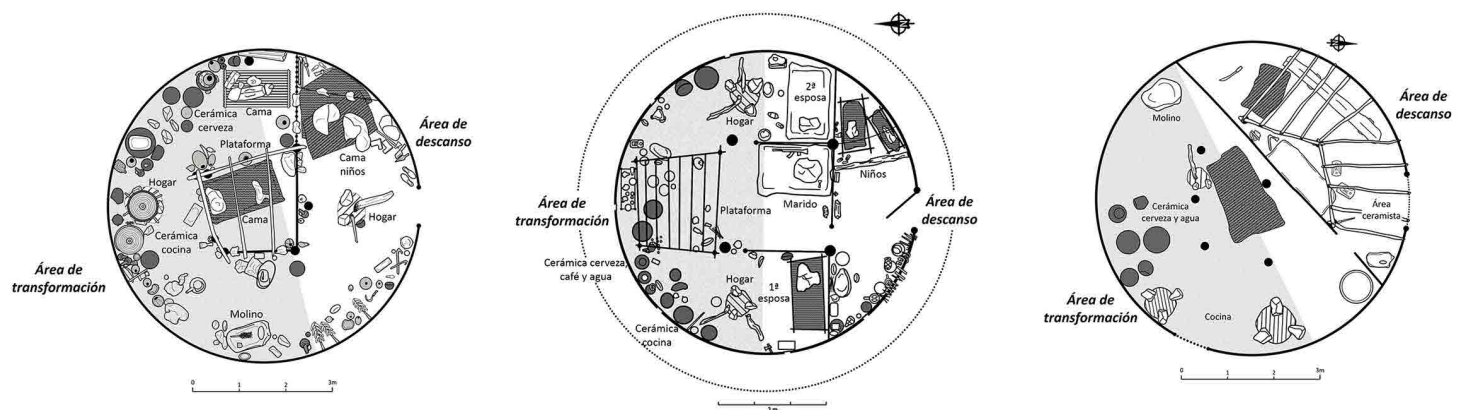

Figura 10. División del espacio interior de las casas gwama-kwama (Tongo-Begi special wereda): Zebsher (izquierda), poblado de reasentamiento de Keser 2 (centro) y K'wak'eb (derecha). Planos del autor. 


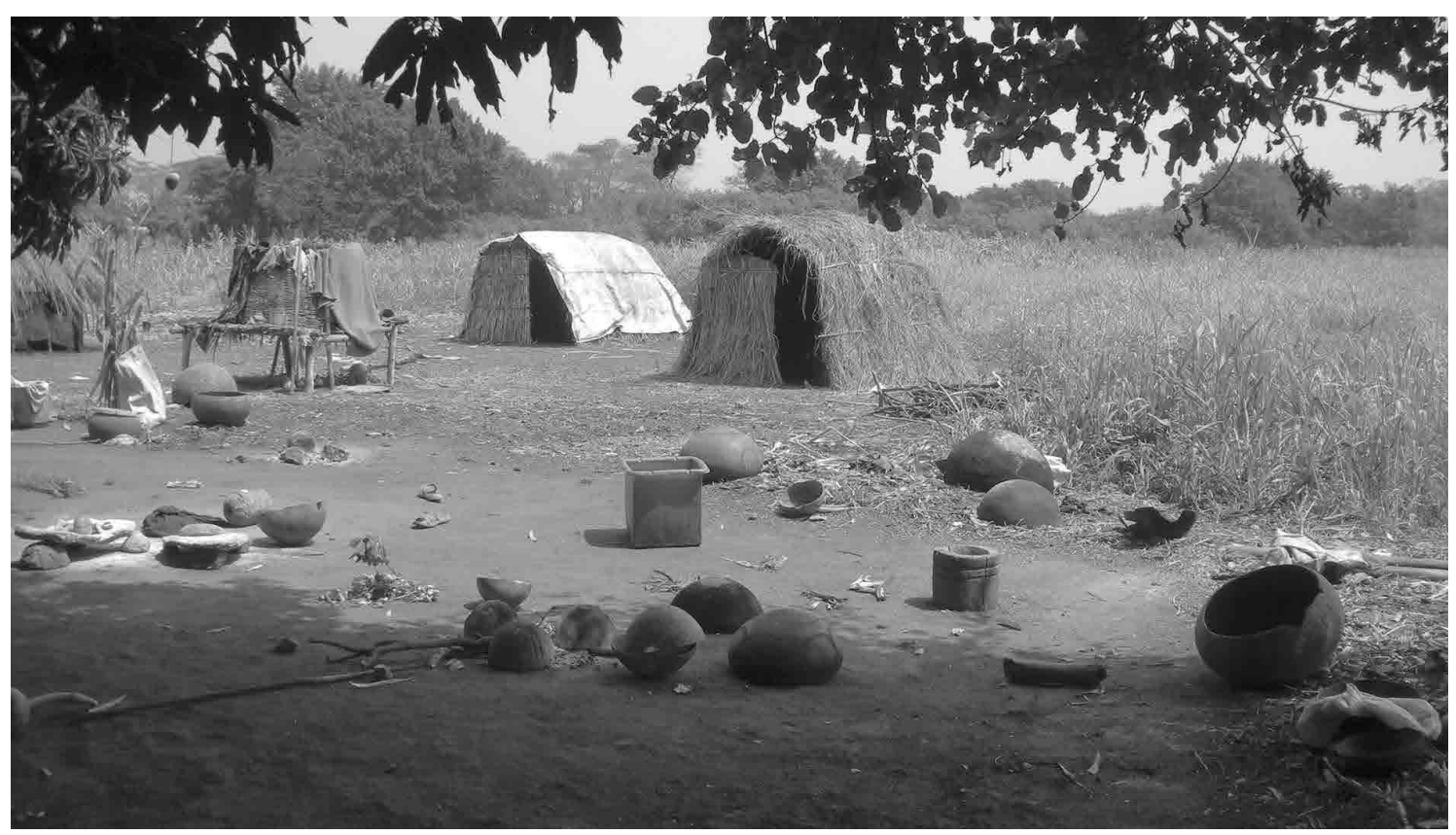

Figura 11. Casa "exteriorizada" opuuo de la aldea de Gwankei (Gambella). Área de cocina en primer plano; área de descanso al fondo. Fotografía de Xurxo Ayán.

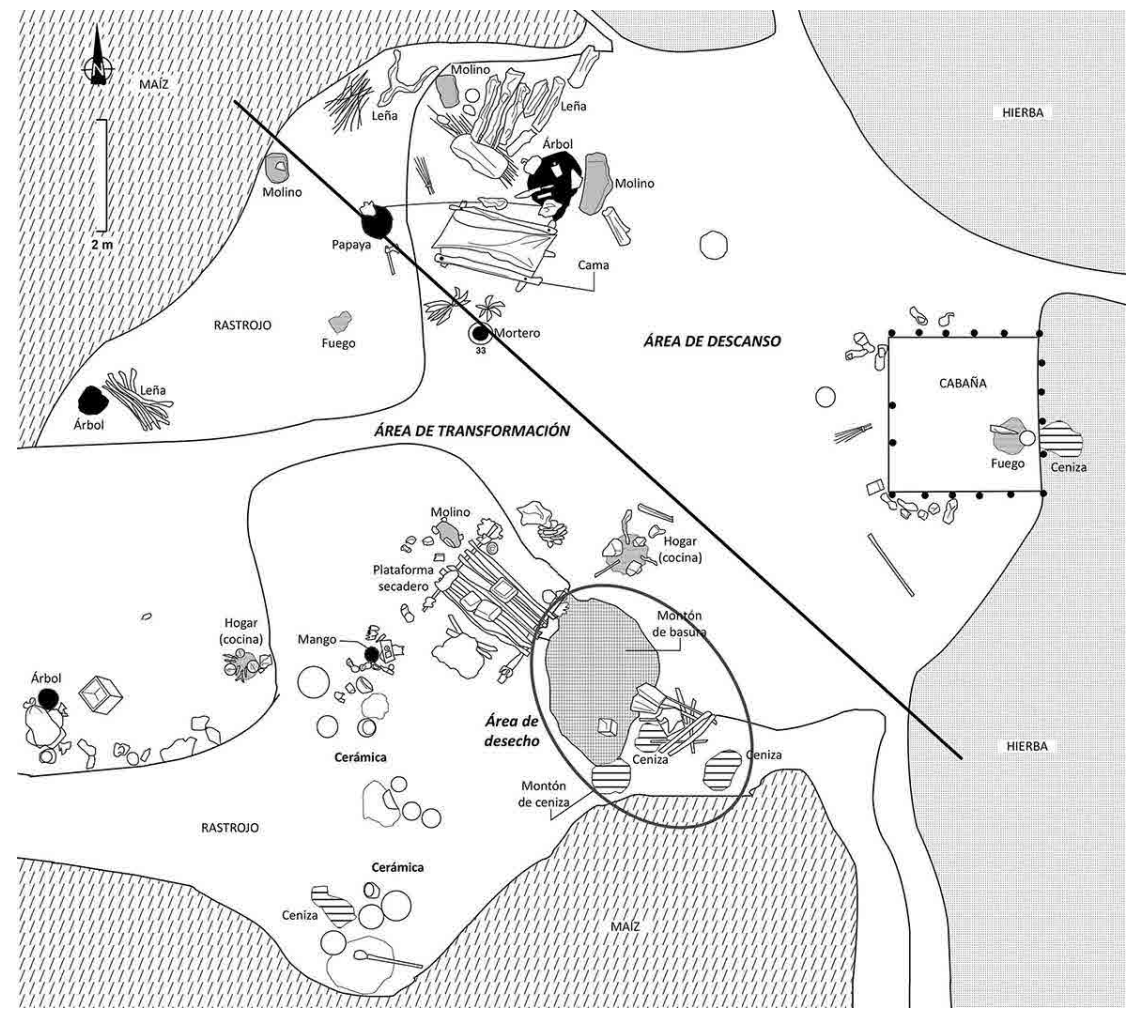

Figura 12. División del espacio doméstico en una casa "exteriorizada" opuuo (Pokong, Gambela), sobre plano de González-Ruibal et al. (2013: fig. 22). 


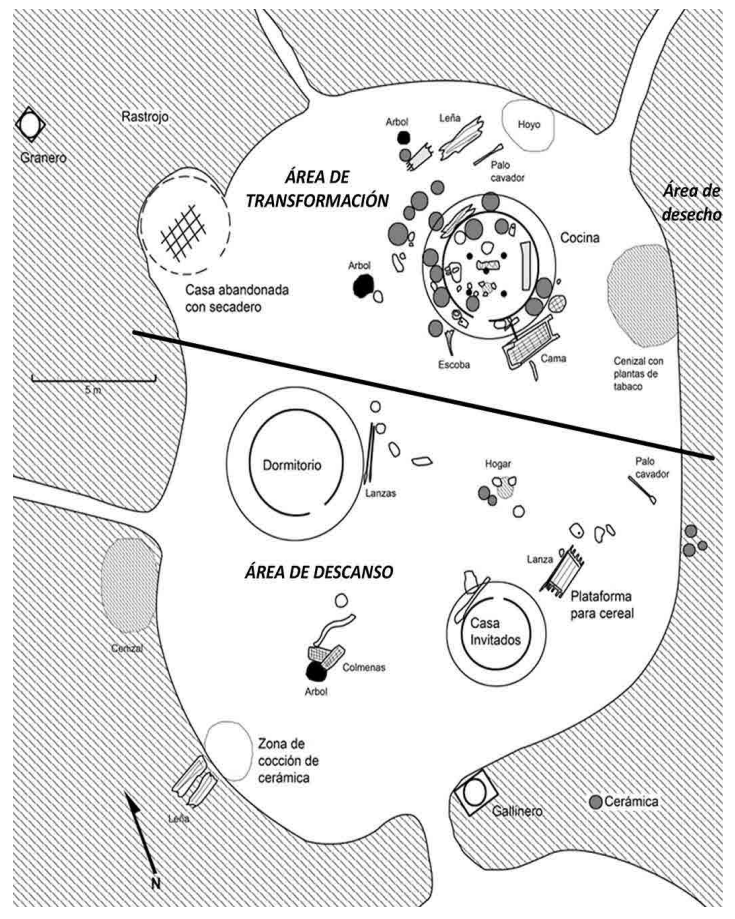

Figura 13. División de las áreas de actividad en una casa komo (Yengu, Tongo-Begi special wereda), sobre plano de González-Ruibal et al. (2013: fig. 14).

condensa las características de la zona exterior comunitaria y el área de descanso propias de los modelos que se han descrito. Del mismo modo, en la parte trasera encontramos el área de transformación, con la estructura que alberga la cocina y todas las cerámicas distribuidas en su interior -tanto las destinadas a preparar la comida como las de la cerveza. Y, en relación con ella, el área de acumulación del desecho, marcada por los grandes cenizales propios de los grupos koman.

Por último, las viviendas gumuz, daats'iin y koman también presentan elementos y características que indican la existencia de un órgano de expulsión o "aparato excretor" de la casa-cuerpo, lo que incide en las propiedades fisiológicas de ésta. Como en el caso bertha, estos elementos están directamente relacionados con el peligro de polución del interior de la casa y de sus habitantes.

Las viviendas gumuz y daats'íin tienen en la parte posterior una abertura en la pared con una gran carga simbólica y práctica, significativamente situada tras las cerámicas para la fermentación de la cerveza. En este caso la abertura toma la forma de puerta trasera, que normalmente permanece cerrada (Fig. 14).
Sólo se abre con ocasión de grandes amenazas, normalmente expresadas por nuestros informantes en términos de ataques desde el exterior del poblado. Explican que esta puerta es una vía de escape ante el peligro de ser capturados como esclavos. En la actualidad estas comunidades ya no corren esos riesgos como lo hacían en el pasado, por lo que la función de la puerta trasera ha quedado como un marcador de la memoria social del desafío a la esclavitud y la guerra por parte del estado (González-Ruibal 2014: 163-64). Los daats'íin dicen, además, que esa puerta se puede abrir en el caso de grandes reuniones de personas, por ejemplo, con ocasión de una boda.

Lo que parece desprenderse de los usos que bertha, gumuz y daats'iin dan a estas aberturas traseras, es el hecho de que su función está relacionada con los peligros de polución e invasión por parte de potencias malignas (visibles e invisibles, humanas y no humanas) del interior de la casa como cuerpo colectivo. Tanto los peligros potenciales que acechan tras el despliegue de las energías sexuales (incluida la menstruación o una boda), como el asalto, captura o muerte de sus habitantes, deben ser regulados por medio de un mecanismo de expulsión, a la vez material, fisiológico y simbólico, que devuelva el equilibrio interno a la casa como cuerpo colectivo.

En el caso de las viviendas koman, no existe ninguna abertura con estas características. Sin embargo, se pone de manifiesto el carácter fisiológico de los procesos y actividades de la casa por el hecho documentado de la práctica tradicional de enterramiento de los intestinos de un fallecido en la parte trasera de la cocina, bajo las cerámicas donde fermenta la cerveza (James 1988: 361). En un mismo sentido puede entenderse la localización de basureros y cenizales -restos de las actividades de transformación de la casa - tras el área de cocina tanto de la vivienda gwama-kwama como de la casa "exteriorizada" komo y opuuo.

En un sentido procesual, antes que clasificatorio y conceptual, la vivienda nilo-sahariana es un espacio fisiológico de gestión y transformación de personas, materiales y substancias. Parece que en todos los casos funciona como un organismo vivo. Al actuar como un cuerpo tiene funciones de comunicación y de descanso, de alimentación y digestivas. También posee funciones sexuales y de expulsión del desecho. Podría decirse que es un cuerpo colectivo en estado de reposo, asentado en el 


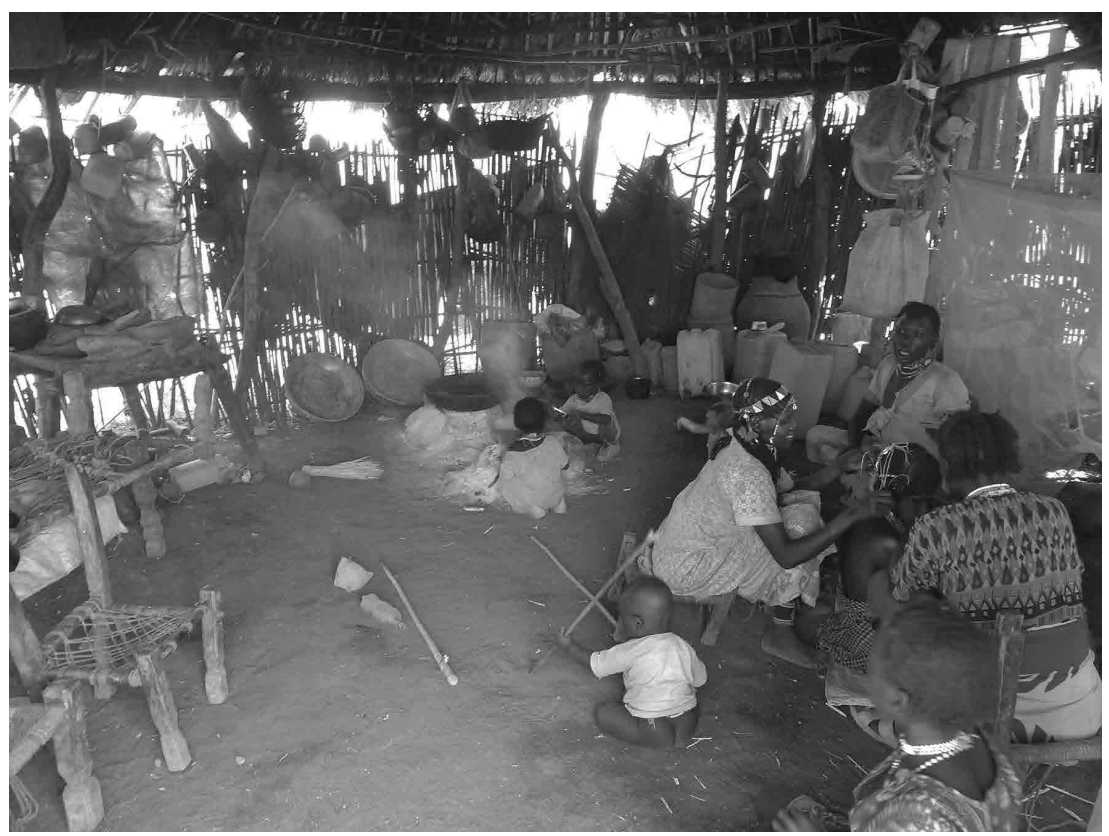

Figura 14. Interior de una casa daats'íin con puerta trasera al fondo, tras las cerámicas para fermentar la cerveza (Mahadid, Qwara). Se aprecian muy bien el "área de descanso", al frente, y el "área de transformación”. Fotografía del autor.

paisaje junto a otros cuerpos con los que comparte relaciones de filiación y vecindad.

Es destacable que en ningún caso la vivienda está generizada, sino que tiene cualidades tanto masculinas como femeninas, pues su agencia trabaja en la producción de una lógica espacial específica que, performativamente, construye sujetos e identidades colectivas en la práctica cotidiana. En todos los grupos, son los hombres los que se hacen cargo de la construcción y mantenimiento de la estructura de la casa (Fig. 15), mientras que las mujeres colaboran en la construcción del tejado y se ocupan de extender, mantener y decorar el manteado de barro de las paredes -entre los gumuz y los bertha-, además de encargarse de las actividades de la cocina de manera exclusiva. De manera general parece que lo masculino se relaciona con los huesos y músculos y lo femenino con las vísceras y la piel, si mantenemos la metáfora de la casa como cuerpo colectivo. Excepto en el caso de la zona de cocina, el resto de las áreas de actividad de la casa, tanto al interior como al exterior, no están tan claramente generizadas, concerniendo tanto a hombres como a mujeres, aunque sí se observa una división de género en cuanto a las funciones y actividades desarrolladas por unas y otros en cada uno de estos contextos. Antes que una división del espacio doméstico según los sexos (del tipo público-exterior-masculino/ privado-doméstico-femenino), parece que lo que se establece en la vivienda es una relación entre sexos, géneros, materiales y substancias. Las mujeres se relacionan, en primer lugar, con las substancias, su modificación y el procesado de su desecho. Pero para realizar estas actividades manejan otro tipo de materiales, como la piedra para los molinos y para los morillos del hogar. Sus actividades también implican el uso de herramientas cortantes, como las azadas para extraer el barro con el que modelar las cerámicas o enlucir las paredes de casas y graneros, o las hachas para hacer leña para el hogar. En cambio, los hombres están relacionados con la elaboración y uso de los elementos de madera, las fibras vegetales y las herramientas y armas cortantes, además de con los soportes de la casa como cuerpo.

De este modo, puede decirse que la casa nilo-sahariana es una casa andrógina, con características tanto masculinas como femeninas (cf. Hugh-Jones 1995).

En definitiva, la casa nilo-sahariana actúa como un cuerpo -no humano sino social, pero humanizado, es decir, destacando su carácter fisiológico, natural- localizado en el espacio y por lo tanto relacionado social y materialmente con el resto de cuerpos sociales que la rodean. Este cuerpo es simultáneamente masculino y 


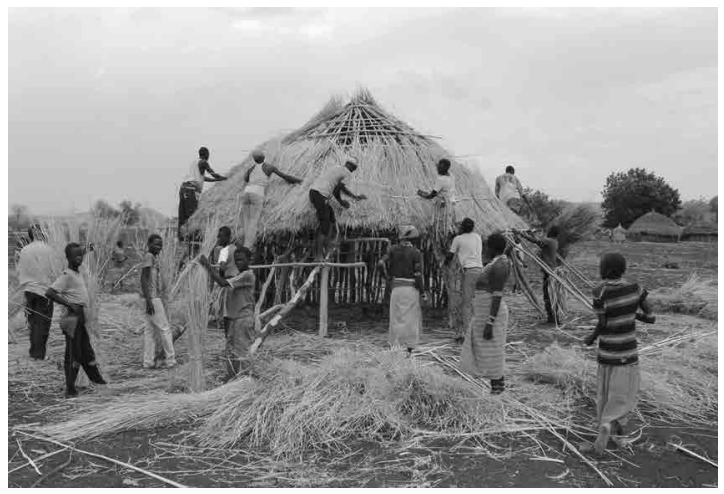

Figura 15. Hombres gumuz poniendo el tejado de una casa en construcción, con la colaboración de las mujeres (Mahal Gerara, Mahadid, Qwara).

Fotografía del autor.

femenino. Se comunica e interactúa comunitariamente en la parte exterior delantera, desarrolla procesos interiores propios del cuerpo -descanso, alimentación y digestión- y deposita sus restos en la parte trasera exterior. El proceso fisiológico de la casa se articula, así, siguiendo un principio de profundidad que relaciona la parte delantera con la trasera a través de un mismo eje, pasando por el interior de la casa-cuerpo. Más que la división en categorías dicotómicas es este eje en profundidad lo que aparece como un principio vertebrador de la acción práctica de la casa como cuerpo colectivo. Si bien es innegable que el espacio doméstico se organiza, además, según un sistema de categorías basado en la dualidad.

\section{Conclusión}

La casa-cuerpo nilo-sahariana se revela como un modelo de casa social, además de ser una unidad residencial familiar. Sus modos de acción, basados en los principios fisiológicos del cuerpo humano, construyen, ordenan y dan sentido al mundo, constituyendo a sus habitantes como un cuerpo colectivo, social. Extendiendo las conclusiones para la casa bertha (González-Ruibal 2006: 399), las casas nilo-saharianas son estructuras estructurantes (Donley-Reid 1990), que distribuyen a los seres que las habitan -vivos y muertos, humanos y cosas-, les orienta y les otorga un lugar en el mundo.

En este sentido, es importante destacar que es el locus donde se ordenan las relaciones sociales de parentesco. Se trata del espacio don- de se materializa una parte fundamental de las relaciones de afinidad entre los segmentos sociales patrilineales que intercambian esposas, puesto que es la residencia del matrimonio. Pero también es el espacio que articula las relaciones de descendencia, puesto que se trata del lugar primario donde se ordenan y clasifican las identidades y funciones de hijos e hijas para dar continuidad a las redes de filiación y de alianza.

Por otro lado, el hecho de que las viviendas nilo-saharianas sean cuerpos colectivos nos habla del carácter participativo de estas sociedades con el entorno que habitan. Como se señalaba en la introducción, las sociedades "primitivas" establecen una actitud participativa con la naturaleza, a diferencia de las sociedades campesinas que tienen una relación de intervención activa en la naturaleza y de transformación del medio. La casa como organismo con propiedades fisiológicas que establece una unidad social basada en el matrimonio, lo que hace es mostrar que "la reproducción social queda íntimamente supeditada a la reproducción natural" (Criado 1995: 93): la vitalidad de la casa-cuerpo, de su descendencia y de la alianza entre afines dependen de la fertilidad de la unión matrimonial.

Finalmente, la casa-cuerpo podría considerarse la materialización de la contradicción esencial de las sociedades nilo-saharianas: al ser sociedades no jerarquizadas, la única división social real es la establecida por el sexo. La casa reúne a ambos sexos bajo el mismo techo y vertebra las relaciones y las actividades siguiendo un principio fisiológico basado en el cuerpo humano, pero acepta una división irresoluble mediante la creación de un sistema dualista de categorías. Este dualismo respondería al núcleo fundamental del antagonismo social dentro de estas sociedades (vid. Žižek 2003: 36-38), unificado por el sistema fisiológico de agencias de la casa-cuerpo como principio de armonización y equilibrio social. Aunque no exista una relación directa entre las divisiones del espacio y la división sexual del trabajo social, la casa-cuerpo nilo-sahariana ordena las actividades y las prácticas técnicas de cada uno de los sexos en cada una de las áreas en que se divide el espacio doméstico, produciendo un habitus material generizado (vid. Lyons 2009: 139-140), situándolos en el mundo y señalando las funciones específicas de cada uno en la organización social y política de estas sociedades "salvajes". 


\section{Agradecimientos}

Los datos en los que se basa este texto han sido recogidos colectivamente en once campañas de trabajo de campo en el occidente de Etiopía desde el año 2002, dentro de diversos proyectos dirigidos por Víctor M. Fernández Martínez y Alfredo González-Ruibal. A ambos agradezco el ánimo, la orientación y la discusión en el estudio monográfico del entorno construido entre las sociedades de la frontera occidental de Etiopía, que dará lugar a la redacción de una tesis doctoral. El registro de los espacios domésticos no podría haberse llevado a cabo sin la participación de Xurxo Ayán. Los acertados comentarios y críticas de Almudena Hernando y de dos revisores anónimos a una primera versión del texto han hecho que este artículo gane en consistencia y claridad. Los errores que puedan apreciarse son responsabilidad exclusiva del autor.

\section{Bibliografía}

Ahland. C. (en prensa): Daats'íin, a newly identified undocumented language of Western Ethiopia: A preliminary examination. Selected Proceedings of the $46^{\text {th }}$ Annual Conference on African Linguistics (Contemporary African Linguistics, 2) (D. L. Payne, S. Pacchiarotti, M. Bosire, eds.), Language Science Press, Berlin.

Clastres, P. (1987): Investigaciones en antropología política. Gedisa, Barcelona.

Criado Boado, F. (1995): Construcción social del espacio y reconstrucción arqueológica del paisaje. La perspectiva espacial en arqueología (C. Barros, J. Nastri, comps.), Centro Editor de América Latina, Buenos Aires: 75-116.

Delmet, Ch. (1974): Notes sur les populations du Djebel Guli (Southern Dar Fung, Soudan). L'Homme, 14(2): 119-134.

Delmet, Ch. (1979): Islamisation et matrilinéarité au Dar Fung (Soudan). L'Homme, 19(2): 33-51.

Donham, D. (1986): Old Abyssinia and the new Ethiopian Empire: Themes in social history. The southern marches of imperial Ethiopia: Essays in history and social anthropology (D. Donham, W. James, eds.), James Currey, Oxford; Ohio University Press, Athens; Addis Ababa University Press, Addis Ababa: 3-48.

Donley-Reid, L. W. (1990): A structuring structure: the Swahili house. Domestic architecture and the use of space (S. Kent, ed.), Cambridge University Press, Cambridge: 114-126.

Ehret, Ch. (2008): Reconstructing Ancient Kinship in Africa. Early Human Kinship. From Sex to Social Reproduction (N. J. Allen, H. Callan, R. Dunbar, W. James, eds.), Blackwell, Oxford: 200-231.

Fanthorpe, R. (1998): Limba 'Deep Rural' Strategies. Journal of African History, 39: 15-38.

Fernández, V. M. (2003): Four thousand years in the Blue Nile. Paths to inequality and ways of resistance. Complutum, 14: 409-425.

Gillespie, S. D. (2007): When is a House? The Durable House: House Society Models in Archaeology (R.A. Beck, ed.), Center for Archaeological Investigations, Occasional Paper No. 35, Southern Illinois University, Carbondale: 25-43.

González-Ruibal, A. (2006): Order in a disordered world. The Bertha house. Anthropos, 101(2): 379-402.

González-Ruibal, A. (2012): Generations of Free Men. Resistance and Material Culture in Western Ethiopia. Beyond Elites. Alternatives to Hierarchical Systems in Modelling Social Formations (T. L. Kienlin, A. Zimmermann, eds.), Dr. Rudolf Habelt GmbH, Bonn: 67-82.

González-Ruibal, A. (2014): An Archaeology of Resistance. Materiality and Time in an African Borderland. Rowman \& Littlefield, Lanham.

González-Ruibal, A.; Ayán, X.; Falquina, A. (2009): Deep-mapping the Gumuz house. Archaeotecture: Second Floor. Papers from the Archaeology of Architecture sessions held at the EAA Meetings in St. Petersburg (2003) and Lyon (2004) (X. Ayán, P. Mañana, R. Blanco, eds.), BAR International Series 1971, Archaeopress, Oxford: 79-96.

González-Ruibal, A.; Ayán, X.; Falquina, A. (2013): Cultura material y etnicidad. Observaciones etnoarqueológicas en la región de Gambela (Etiopía). MATerialidadeS. Perspectivas en cultura material, 1: $57-116$.

Grottanelli, V. L. (1948): I Pre-niloti: un'arcaica provincia culturale in Africa. Annali Lateranensi, 12: 280326.

Haaland, R. (1992): Fish, pots and grain. Early and Mid-Holocene adaptations in the Central Sudan. The African Archaeological Review, 10: 43-64.

Hugh-Jones, S. (1995): Inside-out and back-to-front: the androgynous house in Northwest Amazonia. About the House. Lévi-Strauss and Beyond (J. Carsten, S. Hugh-Jones, eds.), Cambridge University Press, Cambridge: 226-269. 
James, W. (1975): Sister-exchange marriage. Scientific American, 233: 84-94.

James, W. (1980): From aboriginal to frontier society in western Ethiopia. Working papers on society and history in Imperial Ethiopia: The southern periphery from 1880 to 1974 (D. L. Donham, W. James, eds.), African Studies Center-Cambridge University Press, Cambridge: 37-67.

James, W. (1986): Lifelines: Exchange marriage among the Gumuz. The southern marches of imperial Ethiopia (D. Donham, W. James, eds.), Cambridge University Press, Cambridge: 119-147.

James, W. (1988): The Listening Ebony. Moral Knowledge, Religion and Power among the Uduk of Sudan. Clarendon, Oxford.

James, W. (1993): Matrifocus on African women. Defining females. The nature of women in society (S. Ardener, ed.), Berg, Oxford: 123-145.

Jedrej, C. (1995): Ingessana: The religious institutions of a people of the Sudan-Ethiopian borderland. Brill, Leiden.

Jedrej, C. (2004): The Southern Funj of the Sudan as a frontier society, 1820-1980. Comparative Studies in Society and History, 46(4): 709-729.

Last, M. (1979): Some Economic Aspects of Conversion in Hausaland. Conversion to Islam (N. Levtzion, ed.), Homes and Meier, New York.

Lévi-Strauss, C. (1997 [1964]): El pensamiento salvaje. Fondo de Cultura Económica, Santafé de Bogotá.

Lyons, D. E. (2009): How I Built My House. An Ethnoarchaeological Study of Gendered Technical Practice in Tigray, Ethiopia. Ethnoarchaeology, 1(2): 137-161.

Mabogunje, A. L.; Richards, P. (1985): The People and Land of West Africa. History of West Africa (J.F.A. Ajayi, M. Crowder, eds.), Longman, London.

Morgan, L. H. (1987 [1877]): La Sociedad Primitiva. Endymion, Madrid.

Warnier, J-P. (2006): Inside and Outside. Surfaces and Containers. Handbook of Material Culture (C. Tilley, W. Keane, S. Küchler, M. Rowlands, P. Spyer, eds.), SAGE Publications, London-Thousand Oaks-New Delhi: 186-195.

Wolf, E. R. (1975, [1966]): Los campesinos. Lábor, Barcelona.

Žižek, S. (2003): Introducción. El espectro de la ideología. Ideología. Un mapa de la cuestión (S. Žižek, comp.), Fondo de Cultura Económica, Buenos Aires: 7-42. 